\title{
Testosterone Administration Alters Hepatic Blood Flow Across Age: Systematic Review of Animal Experimental Studies
}

\author{
Ricardo Aparecido Baptista Nucci ${ }^{1,2}$ Valéria Simões Tanasov ${ }^{2}$ Walter Krause Neto ${ }^{3}$ \\ Romeu Rodrigues de Souza2,3 Eliane Florencio Gama ${ }^{1,2,3}$
}

${ }^{1}$ Aging Studies Program (MS), Universidade São Judas Tadeu, São Paulo, SP, Brazil

${ }^{2}$ Laboratory of Morphoquantitative Studies and Immunohistochemistry, Department of Aging Sciences, Universidade São Judas Tadeu, São Paulo, SP, Brazil

${ }^{3}$ Laboratory of Morphoquantitative Studies and Immunohistochemistry, Department of Physical Education, Universidade São Judas Tadeu, São Paulo, SP, Brazil

\begin{abstract}
Address for correspondence Ricardo Aparecido Baptista Nucci, PhD Laboratório de Estudos Morfoquantitativos e Imuno-histoquímica, Departmento de Ciências do Envelhecimento, Universidade São Judas Tadeu, Rua Taquari, 546, CEP 03166-000, Mooca, São Paulo, SP, Brazil (e-mail: r.aparecido.nucci@uol.com.br).
\end{abstract}

J Morphol Sci 2018;35:96-101.

\begin{abstract}
Keywords

- aging

- liver

- hepatic structure

- testosterone

- androgenic anabolic steroids

- review

Testosterone levels decline gradually with advancing age. At this point, testosterone administration as a therapy has been largely used to improve different outcomes. However, testosterone induces dose-dependent negative effects on the structure and function of the liver across age. Therefore, the present study aimed to carry out a systematic review of the effects of testosterone administration on the hepatic structure of adult and aged animals. We have searched the PubMed, Google Scholar, Science Direct, Scielo and Lilacs databases for animal experimental studies on the effects of testosterone administration on the hepatic structure across age. After applying the inclusion and exclusion criteria, we included two articles in a systematic review and meta-analysis (regarding adult/aged rats). We have shown through a systematic review with meta-analysis that testosterone may cause chronic hepatic congestion. However, the present review had a small number of studies, which was considered a limitation. At this point, we encourage the development of more studies to elucidate the cellular and molecular mechanisms involved in hepatic injuries, as well as the hepatic metabolism of exogenous testosterone across age.
\end{abstract}

\section{Introduction}

After the second and third decades of life, testosterone levels decline gradually with advancing age, without a clear inflection point or andropause. ${ }^{1}$

The age-related decline in testosterone levels has been associated with reductions in muscle mass, strength, physical function, and libido. ${ }^{2-5}$

At this point, testosterone administration as a therapy has been largely used to improve muscle mass, physical function, mobility, and frailty. ${ }^{1,6-10}$

received

April 6, 2017

accepted

August 3, 2018

published online

August 31, 2018
However, for several reasons, older men can be at a higher risk of experiencing adverse events during testosterone therapy when compared with young men. The plasma clearance of testosterone is lower in men aged $\geq 59$ years than in young men (aged $19-35$ years), ${ }^{11}$ resulting in higher testosterone levels in older men at any testosterone dose.

Some adverse events associated with testosterone administration are prostate-related effects, and cardiovascular-related events. ${ }^{12-14}$ Furthermore, testosterone induces dose-dependent

Copyright @ 2018 by Thieme Revinter

Publicações Ltda, Rio de Janeiro, Brazil
License terms

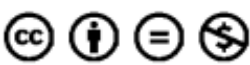


negative effects on the structure and function of the liver across age. $^{15-17}$

The liver has its functions changed with advancing age, resulting in impaired regeneration and in a decrease in drug metabolism. ${ }^{18,19}$ Thus, testosterone administration can induce pathological conditions across age. ${ }^{15,16}$

Currently, the clinical literature uses systematic reviews and meta-analyses to identify possible methodological differences in the studies, in the quality of the surveyed studies on a given topic, and in the most suitable intervention for a specific treatment. ${ }^{8}$ Therefore, the present study aimed to carry out a systematic review of the effects of testosterone administration on the hepatic structure of adult and aged animals.

\section{Materials and Methods}

The present systematic review was developed based on the preferred reporting items for systematic reviews and metaanalyses (PRISMA) guideline, ${ }^{20}$ which is currently used for clinical studies. Therefore, Krause Neto and Nucci adapted it for this research.

On February, 2017, we performed a systematic review search in the PubMed, Google Scholar, Science Direct, Scielo and Lilacs databases, using the following Medical Subject Headings (MeSH) and entry terms: Liver OR Livers OR Bile Ducts; Intrahepatic OR Bile Duct; Intrahepatic OR Duct; Intrahepatic Bile OR Ducts; Intrahepatic Bile OR Intrahepatic Bile Duct OR Intrahepatic Bile Ducts OR Hepatocytes OR Hepatocyte OR Hepatic Cells OR Cell; Hepatic OR Cells; Hepatic OR Hepatic Cell OR Hep G2 Cells OR Cell; Hep G2 OR Cells; Hep G2 OR Hep G2 Cell OR HepG2 Cells OR Cell; HepG2 OR Cells; HepG2 OR HepG2 Cell OR Hep G2 Cell Line OR Hepatoblastoma G2 Cell Line OR Cell Line; Hep G2 OR Cell Line; Hepatoblastoma G2 OR Kupffer Cells OR Cells; Kupffer OR Hepatic Stellate Cells OR Cell; Hepatic Stellate OR Cells; Hepatic Stellate OR Hepatic Stellate Cell OR Stellate Cell; Hepatic OR Stellate Cells; Hepatic OR Ito Cells OR Cells; Ito OR Drug-Induced Liver Injury; Chronic OR Drug Induced Liver Injury; Chronic OR Liver Injury; DrugInduced, Chronic OR Chronic Drug-Induced Liver Injury OR Chronic Drug Induced Liver Injury; AND testosterone OR 17beta-Hydroxy-4-Androsten-3-one OR 17 beta hydroxy 4 androsten 3 one OR androtropy OR dr. kade brand of testosterone OR histone OR hauck brand of testosterone OR stereotaxy OR ulmer brand of testosterone OR sustain OR endoderm OR watson brand of testosterone OR faulding brand of testosterone OR paladin brand of testosterone OR astrazeneca brand of testosterone OR cepa brand of testosterone OR testoderm OR ortho brand of testosterone OR ferring brand of testosterone OR testolin OR pasadena brand of testosterone OR testopel OR barton brand of testosterone OR testosterone sulfate OR androgen OR solvay brand of testosterone OR univmed brand of testosterone OR schering brand of testosterone OR 8-Isotestosterone OR 8 isotestosterone OR 17-beta-Hydroxy-8 alpha-4-Androsten-3-one OR 17 beta hydroxy 8 alpha 4 androsten 3 one $\mathrm{OR}$ andropatch $\mathrm{OR}$ smithkline beecham brand of testosterone OR glaxosmithkline brand of testosterone OR testis OR auxilin pharmaceuticals inc. brand of testosterone OR testosterone propionate OR testosteronpropionat eifelfango
OR propionate eifelfango, testosterone OR eifelfango brand of testosterone propionate OR virormone OR ferring brand of testosterone propionate OR agovirin OR testosterone 17 betacypionate OR testosterone 17 beta-cyclopentylpropionate OR testosterone cypionate OR testosterone 17 beta cyclopentanepropionate OR depo-testosterone OR pfizer brand of testosterone 17 beta-cypionate OR depo-testosterone cypionate OR leposternon OR duarte $s$ OR roberts brand of testosterone 17 beta cypionate OR testa-c OR vojtech brand of testosterone 17 beta-cypionate OR tested elmu OR byk brand of testosterone 17 beta-cypionate OR andronati OR pasadena brand of testosterone 17 beta-cypionate OR depostomead OR spencer mead brand of testosterone 17 beta-cypionate OR testosterone enanthate OR testosterone heptylate OR testosterone heptanoate $\mathrm{OR}$ delatestryl OR theramex brand of testosterone enanthate $\mathrm{OR}$ btg brand of testosterone enanthate OR duraphat $\mathrm{OR}$ roberts brand of testosterone enanthate $\mathrm{OR}$ theramex OR testosterone depot rotexmedica OR rotexmedica brand of testosterone enanthate OR testosterone depot eifelfango OR eifelfango brand of testosterone enanthate OR testosterone depot jenapharm OR jenapharm brand of testosterone enanthate OR westrin p.a. OR pasadena brand of testosterone enanthate OR and repository OR rugby brand of testosterone enanthate OR primoteston depot OR schering brand of testosterone enanthate OR testosterone-17-succinate OR testosterone hydrogen succinate OR testosterone-17hemisuccinate OR T-17-HS OR testosterone hemisuccinate OR testosterone-17-succinate, sodium salt, (17beta) isomer OR testosterone-17-sulfate OR testosterone 17-sulphate OR testosterone-17-sulfate, sodium salt OR testosterone-17-sulfate, ammonium salt OR testosterone-17-sulfate, (17alpha) isomer OR testosterone undecanoate OR testosterone undecylate OR nebido OR understory OR andriole OR batstone OR restandol OR organon brand of testosterone undecanoate OR methyltestosterone OR 17 beta-Hydroxy-17-methyl-4-androsten-3-one OR 17 beta hydroxy 17 methyl 4 androsten 3 one OR 17beta methyltestosterone OR 17beta methyltestosterone OR 17-Epimethyltestosterone OR 17 epimethyltestosterone OR 17betaHydroxy-17-methyl-4-androsten-3-one OR 17beta hydroxy 17 methyl 4 androsten 3 one OR 17 beta methyltestosterone OR 17 beta-methyltestosterone OR android OR in band 1 of methyltestosterone OR Android-10 OR android 10 OR Android-25 OR android 25 OR Android-5 OR android 5 OR western OR mesterolone OR math test OR global pharmaceutical brand of methyltestosterone OR oregon OR schering brand of methyltestosterone OR testoviron OR tested OR in band 2 of methyltestosterone OR virion OR star brand of methyltestosterone OR 17alpha-Methyltestosterone OR 17 alpha OR methyltestosterone OR 17alpha-Methyltestosterone OR 17alpha methyltestosterone OR 17alpha-MethylTestosterone OR 17alpha methyl testosterone OR perandren OR testosterone 17-phenylpropionate OR testosterone phenylpropionate OR restandol OR testosterone decanoate $\mathrm{OR}$ testosterone replacement $\mathrm{OR}$ androgenic anabolic steroids OR anabolic steroids AND Aging OR Senescence OR Biological Aging OR Aging, Biological OR aged OR elderly OR Frail Elderly OR Elderly, Frail OR Frail Elders OR Elder, Frail OR Elders, Frail OR Frail Elder OR FunctionallyImpaired Elderly OR Elderly, Functionally-Impaired OR 
Functionally Impaired Elderly OR Frail Older Adults OR Adult, Frail Older OR Adults, Frail Older OR Frail Older Adult OR Older Adult, Frail OR Older Adults, Frail.

\section{Inclusion and Exclusion Criteria}

We have searched for animal experimental design studies on the administration of testosterone on the hepatic structure and the effect of aging on it. The inclusion criteria were articles with healthy young, adult or aged rodents, testosterone administration, and detailed data on hepatic morphology. We have excluded all of the papers that investigated testosterone administration on genetically modified animals, interventions such as surgery, or the use of any drug or nutritional supplement combined with testosterone administration. The strain of the rodents was not stated as an inclusion criterion.

\section{Outcomes}

The outcomes of interest were the area and the number of nuclei of hepatocytes, which indicate mitosis related to hepatic injury, as well as lobular (capillaries sinusoids, perisinusoidal spaces, and biliary ducts) and non-lobular (portal spaces, veins, and center-lobular branches of blood vessels) parenchyma components, which indicate changes in the hepatic blood flow.

\section{Data Extraction and Analysis of Data Quality}

We have extracted data about the strain of the rodents, as well as their age, gender, testosterone administration dosage and duration, and primary outcomes. Usually, clinical systematic review researchers apply a data quality analysis on the methodology of the analyzed papers through specific topic questionnaires. However, questionnaires for these purposes do not exist for animal studies. Therefore, we did not apply any data quality analysis in the present study. Data such as strain and/or age of the rodents were not used in the search strategy so as to not limit the amount of included studies.

\section{Data Synthesis and Analysis}

The systematic review data was organized in - Tables 1 and 2. ${ }^{15,16}$ For the meta-analysis, RevMan 5.2 software (The Nordic Cochrane Centre, Copenhagen, Denmark) was used to calculate heterogeneity by the i-squared $\left(\mathrm{I}^{2}\right)$ and chisquared $\left(\mathrm{X}^{2}\right)$ values. We have used the $\mathrm{I}^{2}$ to assess the heterogeneity/homogeneity between the studies, using fixed effect models. We have also used the inverse variance method and 95\% total confidence interval (95\%CI).

The present study is an analysis of published data; thus, it did not require the approval of an ethics committee.

\section{Results}

\section{Selected Studies}

After the initial intersection of mesh terms, entry terms, and/ or related keywords, the search identified 12,924 article titles. From this point, 4 evaluators (Nucci, Tanasov, de Souza and Gama) read the titles. Then, the abstracts were selected following the PRISMA ${ }^{20}$ guideline. Of the total 12,924 articles, 52 were used for the initial abstract analysis. The abstracts should contain sufficient data on the parameters of the hepatic structure, on the animals studied, and on the treatments and/or interventions used in the studies. From these, five articles were included for full text analysis. After the inclusion and exclusion criteria were applied, two papers were included in the systematic review and meta-analysis. $^{15,16}$ However, the small number of included papers should be considered a limitation.

\section{Animal Strain, Age and Gender}

In the selected articles, the strain of rodents used was Wistar rats (Rattus norvegicus). This strain is widely used in aging studies. Both studies presented data about the effects of testosterone administration on the hepatic structure of adult or aged animals. Both studies used male animals.

\section{Testosterone Administration, Duration and Dosage}

Tanasov et al $^{16}$ used testosterone propionate via intramuscular administration ( $3 \mathrm{mg} / \mathrm{kg}$ ) for 3 months. However, Nucci et $\mathrm{al}^{15}$ used testosterone propionate via intraperitoneal administration (10 mg/kg) for 4 months.

\section{Data Synthesis}

Both studies ${ }^{15,16}$ used morphometrical and stereological methods to analyze the area and the number of nuclei of hepatocytes, as well as the lobular and non-lobular parenchyma components.

All data are presented by the analysis of 2 studies, and 24 animals were included. Only adult and aged rodent data was considered. Age effect has not been evaluated at this point because the included articles did not present enough internal statistical data.

\section{Meta-analysis}

\section{Number of Nuclei of Hepatocytes}

The forest plot analysis demonstrated homogeneity ( $p=0.97$; $\mathrm{I}^{2}=0 \%$ between the studies, and the testosterone

Table 1 Data description regarding the strain of the rodents, as well as their age, gender, testosterone dosage, frequency (times/ week) and duration

\begin{tabular}{|l|l|l|l|l|l|l|l|}
\hline Reference & Animal & \multicolumn{3}{l|}{ Age (months) } & Gender & \multicolumn{2}{l|}{ Testosterone } \\
\cline { 3 - 8 } & strain & Before & After & & Dosage & $\begin{array}{l}\text { Frequency } \\
\text { (times/week) }\end{array}$ & $\begin{array}{l}\text { Duration } \\
\text { (months) }\end{array}$ \\
\hline Tanasov et al, $2014^{16}$ & Wistar & 13 & 16 & Male & $3 \mathrm{mg} / \mathrm{kg}$ & 3 & 3 \\
\hline Nucci et al, $2017^{15}$ & Wistar & 20 & 24 & Male & $10 \mathrm{mg} / \mathrm{kg}$ & 2 & 4 \\
\hline
\end{tabular}


Testosterone Administration Alters Hepatic Blood Flow Across Age Nucci et al. 99

Table 2 Summary of primary outcomes regarding the area and the number of nuclei of hepatocytes, and of lobular and non-lobular parenchyma components in the groups that received testosterone

\begin{tabular}{|l|l|l|l|l|}
\hline \multirow{2}{*}{ Reference } & \multicolumn{2}{|l|}{ Hepacotyces nuclei } & \multicolumn{3}{l|}{ Parenchyma components } \\
\cline { 2 - 5 } & Number & Area & Lobular & Non-lobular \\
\hline Tanasov et al, $2014^{16}$ & Increased & Decreased & Increased & Decreased \\
\hline Nucci et al, $2017^{15}$ & Increased & Decreased & Increased & Decreased \\
\hline
\end{tabular}

administration had a non-significant $(p=0.15)$ increase of 3.23 hepatocyte nuclei per field (95\% CI: -1.15-7.61). - Fig. 1 presents the data and the forest plot.

\section{Area of Nuclei of Hepatocytes}

The forest plot analysis demonstrated homogeneity ( $p=0.56 ; \mathrm{I}^{2}=0 \%$ ) between the studies, and the testosterone administration had a non-significant ( $p=0.52$ ) decrease of $2.44 \mu \mathrm{m}^{2}$ in the hepatocyte nuclei area (95\% CI: $-9.90-$ 5.01). - Fig. 2 presents the data and the forest plot.

\section{Lobular Parenchyma Components}

The forest plot analysis demonstrated heterogeneity $\left(p=0.04 ; \mathrm{I}^{2}=77 \%\right)$ between the studies, and the testosterone administration had a significant $(p=0.0008)$ increase of $5.77 \%$ in the lobular parenchyma components (95\% CI: $2.40-$ 9.15). - Fig. 3 presents the data and the forest plot.

\section{Non-lobular Parenchyma Components}

The forest plot analysis demonstrated homogeneity ( $p=0.98 ; \mathrm{I}^{2}=0 \%$ ) between the studies, and the testosterone administration had a non-significant ( $p=0.69$ ) decrease of $1.21 \%$ of in the non-lobular parenchyma components ( $95 \%$ CI: -7.12-4.69). - Fig. 4 presents the data and the forest plot.

\section{Discussion}

Through a systematic review with meta-analysis, the present article analyzed the effects of testosterone administration on the hepatic structure of adult and aged animals. We have shown through a meta-analysis that testosterone may increase the mitotic capacity of the hepatocytes. The merging of both studies showed a decrease in the hepatocyte nuclei area, as well as an increase in the number of hepatocyte nuclei per field. This is consistent with studies that highlighted the role of testosterone

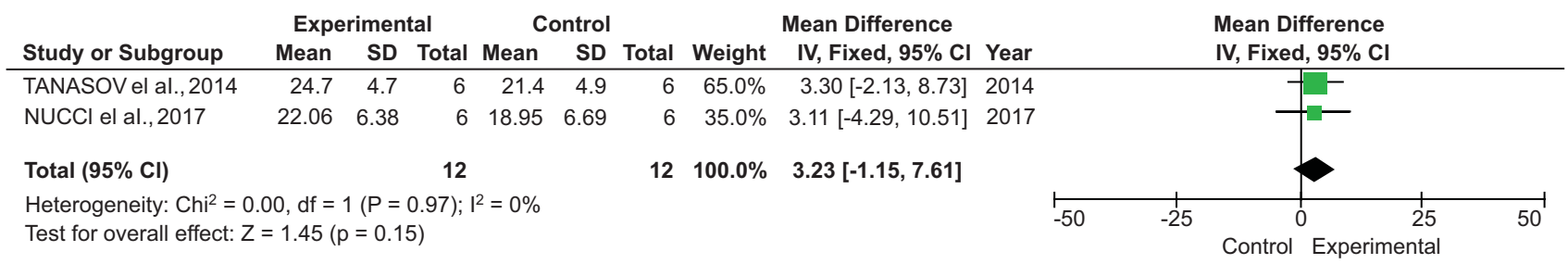

Fig. 1 Forest plot presenting information about the effects of testosterone administration on the number of nuclei of hepatocytes in adult and aged rats (figure created using theRevMan 5.2 2017)

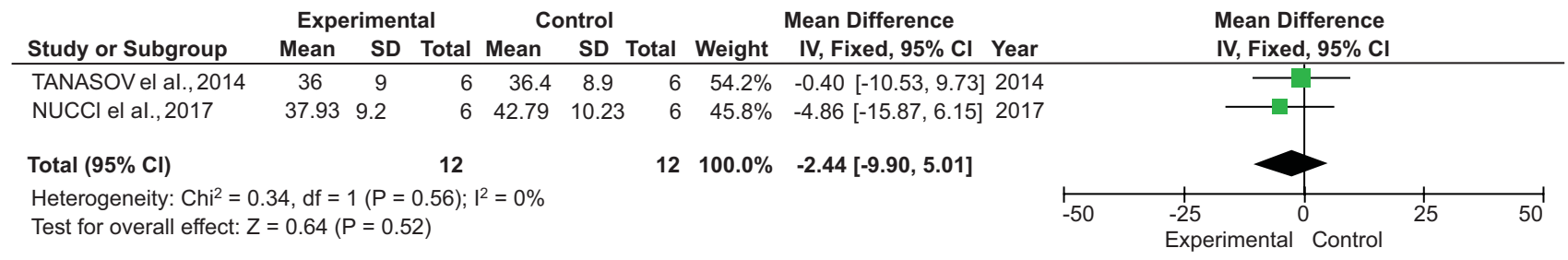

Fig. 2 Forest plot presenting information about the effects of testosterone administration on the area of nuclei of hepatocytes in adult and aged rats (figure created using the RevMan 5.2 2017)

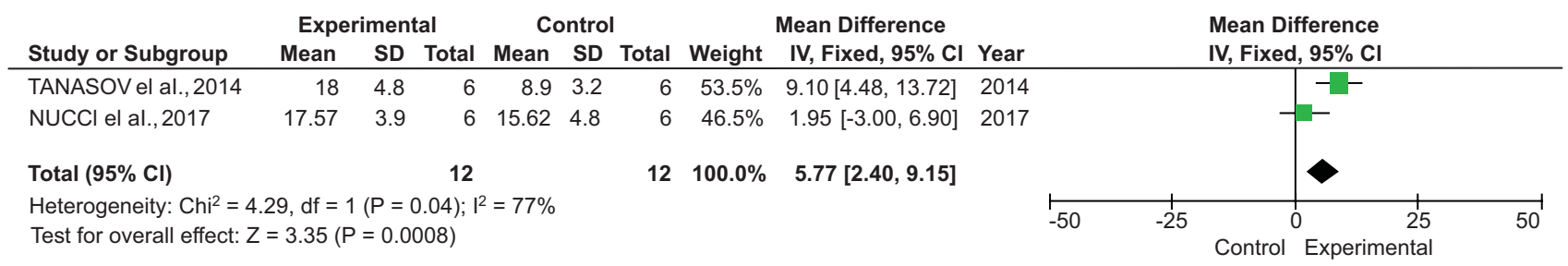

Fig. 3 Forest plot presenting information about the effects of testosterone administration on the lobular parenchyma components in adult and aged rats (figure created using the RevMan 5.2 2017) 


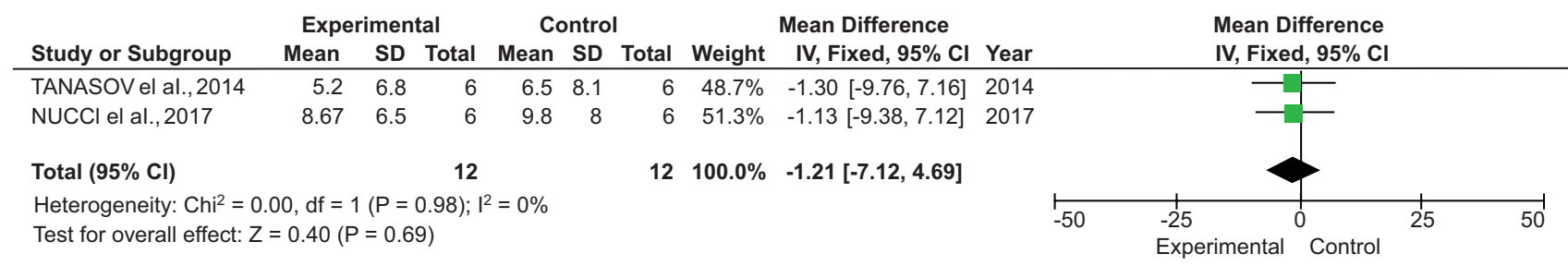

Fig. 4 Forest plot presenting information about the effects of testosterone administration on the non-lobular parenchyma components in adult and aged rats (figure created using the RevMan 5.2 2017)

as a contributing factor in mitosis due to its chemical and toxic aggression to the hepatic tissue. ${ }^{15-17,22}$

However, our major finding was that testosterone changed the hepatic blood flow by increasing the lobular parenchyma components in both studies. ${ }^{15,16}$ Testosterone, at physiological concentrations, can selectively suppress the entry of calcium ions $\left(\mathrm{Ca}^{2+}\right)$ via L-type $\mathrm{Ca}^{2+}$ channels, which indicates its vasodilatory actions. ${ }^{23}$ Nonetheless, with testosterone administration, its vasodilatory actions could be more negative to the hepatic tissue in a dose-dependent fashion.

As the hepatic sinusoids dilate to accommodate the backflow of blood, the liver becomes tensely swollen. ${ }^{24}$ Hence, this process may lead to blood stasis in the non-lobular parenchyma, leading to a hypoxic state. At this moment, a pathological process named hepatic congestion is characterized. ${ }^{15,24,25}$

In addition, Simonetto et $\mathrm{al}^{26}$ demonstrated through an experimental study in murine that chronic hepatic congestion leads to sinusoidal thrombosis and strain, which in turn promote hepatic fibrosis in the long-term. Additionally, a previous autopsy study of livers from patients with congestive heart failure demonstrated sinusoidal thrombi in the proximity of areas with fibrosis, and suggested a causative role of intrahepatic thrombosis in the incidence of fibrosis. ${ }^{27}$ Furthermore, Nucci et al $^{15}$ showed that testosterone can injure the liver tissue, resulting in a sharp increase in fibrosis, which indicates chronic hepatic congestion in the short-term.

\section{Conclusion}

We have shown through a systematic review with metaanalysis that testosterone may cause chronic hepatic congestion in the short-term. However, the present review analyzed a small number of studies, which was considered a limitation. At this point, we encourage the development of more studies to elucidate the cellular and molecular mechanisms involved in hepatic injuries, as well as the hepatic metabolism of exogenous testosterone across age.

\section{References}

1 Spitzer M, Huang G, Basaria S, Travison TG, Bhasin S. Risks and benefits of testosterone therapy in older men. Nat Rev Endocrinol 2013;9(07):414-424 Doi: 10.1038/nrendo.2013.73

2 Orwoll E, Lambert LC, Marshall LM, et al; Osteoporotic Fractures in Men Study Group. Endogenous testosterone levels, physical performance, and fall risk in older men. Arch Intern Med 2006; 166(19):2124-2131 Doi: 10.1001/archinte.166.19.2124
3 Roy TA, Blackman MR, Harman SM, Tobin JD, Schrager M, Metter EJ Interrelationships of serum testosterone and free testosterone index with FFM and strength in aging men. Am J Physiol Endocrinol Metab 2002;283(02):E284-E294 Doi: 10.1152/ajpendo.00334.2001

4 Travison TG, Morley JE, Araujo AB, O'Donnell AB, McKinlay JB. The relationship between libido and testosterone levels in aging men. J Clin Endocrinol Metab 2006;91(07):2509-2513 Doi: 10.1210/ jc.2005-2508

5 van den Beld AW, de Jong FH, Grobbee DE, Pols HA, Lamberts SW. Measures of bioavailable serum testosterone and estradiol and their relationships with muscle strength, bone density, and body composition in elderly men.J Clin Endocrinol Metab 2000;85(09): 3276-3282 Doi: 10.1210/jcem.85.9.6825

6 Ferrando AA, Sheffield-Moore M, Yeckel CW, et al. Testosterone administration to older men improves muscle function: molecular and physiological mechanisms. Am J Physiol Endocrinol Metab 2002;282(03):E601-E607 Doi: 10.1152/ajpendo.00362.2001

7 Kenny AM, Prestwood KM, Gruman CA, Marcello KM, Raisz LG. Effects of transdermal testosterone on bone and muscle in older men with low bioavailable testosterone levels. J Gerontol A Biol Sci Med Sci 2001;56(05):M266-M272 Doi: 10.1093/gerona/56.5.M266

8 Krause Neto W, Ciena AP, Anaruma CA, de Souza RR, Gama EF. Effects of exercise on neuromuscular junction components across age: systematic review of animal experimental studies. BMC Res Notes 2015;8:713 Doi: 10.1186/s13104-015-1644-4

9 Page ST, Amory JK, Bowman FD, et al. Exogenous testosterone(T)alone or with finasteride increases physical performance, grip strength, and lean body mass in older men with low serum T. J Clin Endocrinol Metab 2005;90(03):1502-1510 Doi: 10.1210/jc.2004-1933

10 Sih R, Morley JE, Kaiser FE, Perry HM III, Patrick P, Ross C. Testosterone replacement in older hypogonadal men: a 12month randomized controlled trial. J Clin Endocrinol Metab 1997;82(06):1661-1667 Doi: 10.1210/jcem.82.6.3988

11 Coviello AD, Lakshman K, Mazer NA, Bhasin S. Differences in the apparent metabolic clearance rate of testosterone in young and older men with gonadotropin suppression receiving graded doses of testosterone. J Clin Endocrinol Metab 2006;91(11):4669-4675 Doi: $10.1210 /$ jc.2006-0822

12 Bhasin S, Cunningham GR, Hayes FJ, et al; Task Force, Endocrine Society. Testosterone therapy in men with androgen deficiency syndromes: an Endocrine Society clinical practice guideline. J Clin Endocrinol Metab 2010;95(06):2536-2559 Doi: 10.1210/ jc.2009-2354

13 Calof OM, Singh AB, Lee ML, et al. Adverse events associated with testosterone replacement in middle-aged and older men: a metaanalysis of randomized, placebo-controlled trials. J Gerontol A Biol Sci Med Sci 2005;60(11):1451-1457 Doi: 10.1093/gerona/ 60.11 .1451

14 Xu L, Freeman G, Cowling BJ, Schooling CM. Testosterone therapy and cardiovascular events among men: a systematic review and meta-analysis of placebo-controlled randomized trials. BMC Med 2013;11(01):108 Doi: 10.1186/1741-7015-11-108

15 Nucci RAB, Teodoro ACS, Krause Neto W, et al. Effects of testosterone administration on liver structure and function in aging rats. Aging Male 2017;20(02):134-137 Doi: 10.1080/13685538.2017.1284779 
16 Tanasov VS, Krause Neto W, Gonçalves L, Maifrino LBM, De Sousa RR, Gama EF. Use of Anabolic Steroid Altered the Liver Morphology of Rats. Int J Morphol 2014;32(03):756-760 Doi: 10.4067| S0717-95022014000300002

17 Vieira RP, França RF, Damaceno-Rodrigues NR, et al. Dosedependent hepatic response to subchronic administration of nandrolone decanoate. Med Sci Sports Exerc 2008;40(05): 842-847

18 Nucci RAB, Teodoro ACS. GAMA EF. Liver regeneration and aging: a review. J Morphol 2016;33(04):179-182 Doi: 10.4322/jms.100616

19 Schmucker DL. Age-related changes in liver structure and function: Implications for disease? Exp Gerontol 2005;40(89):650-659 Doi: 10.1016/j.exger.2005.06.009

20 Liberati A, Altman DG, Tetzlaff J, et al. The PRISMA statement for reporting systematic reviews and meta-analyses of studies that evaluate health care interventions: explanation and elaboration. PLoS Med 2009;6(07):e1000100 Doi: 10.1371/journal. pmed. 1000100

21 Krause Neto W, Gama EF, Rocha LY, et al. Effects of testosterone on lean mass gain in elderly men: systematic review with meta- analysis of controlled and randomized studies. Age (Dordr) 2015; 37(01):9742

22 Michalopoulos GK, DeFrances MC. Liver regeneration. Science 1997;276(5309):60-66

23 Hall J, Jones RD, Jones TH, Channer KS, Peers C. Selective inhibition of L-type $\mathrm{Ca} 2+$ channels in A7r5 cells by physiological levels of testosterone. Endocrinology 2006;147(06):2675-2680 Doi: 10.1210/en.2005-1243

24 Gore RM, Mathieu DG, White EM, Ghahremani GG, Panella JS, Rochester D. Passive hepatic congestion: cross-sectional imaging features. AJR Am J Roentgenol 1994;162(01):71-75 Doi: 10.2214/ ajr.162.1.8273693

$25 \mathrm{Ou} \mathrm{QJ}$, Hermann RE. The role of hepatic veins in liver operations. Surgery 1984;95(04):381-391

26 Simonetto DA, Yang HY, Yin M, et al. Chronic passive venous congestion drives hepatic fibrogenesis via sinusoidal thrombosis and mechanical forces. Hepatology 2015;61(02):648-659

27 Wanless IR, Liu JJ, Butany J. Role of thrombosis in the pathogenesis of congestive hepatic fibrosis (cardiac cirrhosis). Hepatology 1995;21(05):1232-1237 